\title{
Hax1 lacks BH modules and is peripherally associated to heavy membranes: implications for Omi/HtrA2 and PARL activity in the regulation of mitochondrial stress and apoptosis
}

\author{
DV Jeyaraju ${ }^{1}$, G Cisbani ${ }^{1}$, OM De Brito ${ }^{2}$, EV Koonin ${ }^{3}$ and L Pellegrini ${ }^{*, 1,4}$
}

Hax1 has an important role in immunodeficiency syndromes and apoptosis. A recent report (Chao et al., Nature, 2008) proposed that the Bcl-2-family-related protein, Hax1, suppresses apoptosis in lymphocytes and neurons through a mechanism that involves its association to the inner mitochondrial membrane rhomboid protease PARL, to proteolytically activate the serine protease Omi/HtrA2 and eliminate active Bax. This model implies that the control of cell-type sensitivity to pro-apoptotic stimuli is governed by the PARL/Hax1 complex in the mitochondria intermembrane space and, more generally, that Bcl-2-family-related proteins can control mitochondrial outer-membrane permeabilization from inside the mitochondrion. Further, it defines a novel, anti-apoptotic Opa1-independent pathway for PARL. In this study, we present evidence that, in vivo, the activity of Hax1 cannot be mechanistically coupled to PARL because the two proteins are confined in distinct cellular compartments and their interaction in vitro is an artifact. We also show by sequence analysis and secondary structure prediction that Hax 1 is extremely unlikely to be a Bcl-2-family-related protein because it lacks Bcl-2 homology modules. These results indicate a different function and mechanism of Hax 1 in apoptosis and re-opens the question of whether mammalian PARL, in addition to apoptosis, regulates mitochondrial stress response through Omi/HtrA2 processing.

Cell Death and Differentiation (2009) 16, 1622-1629; doi:10.1038/cdd.2009.110; published online 14 August 2009

Mitochondria are key players in central cellular processes, such as ATP production, $\mathrm{Ca}^{2+}$ signaling and apoptosis. Mitochondrial involvement in apoptosis has been thoroughly documented during the last decade. Its two main features include the release of proteins from the mitochondrial intermembrane space (IMS) and the initiation of a program of dysfunction that includes the loss of the proton electrochemical gradient across the inner mitochondrial membrane (IMM). ${ }^{1}$ These two cascades of events seem to be mediated by the crosstalk of several molecular mechanisms that are still not fully characterized. ${ }^{2}$ Nevertheless, the general consensus is that during apoptosis, mitochondria release cytochrome $\mathrm{C}$ and other proteins that cooperate to execute programmed cell death. ${ }^{3-5}$

The function of mitochondria in the regulation and amplification of the apoptotic cascade is regulated by members of the $\mathrm{Bcl}-2$ protein family. ${ }^{6,7}$ These are cytosolic proteins that, under steady state conditions, are mainly peripherally associated to heavy membranes, ${ }^{8}$ and that share a limited structural similarity with $\mathrm{Bcl}-2$ in the so called Bcl-2 homology (BH) module. Bcl-2-family-related proteins participate in the same process of regulation of apoptosis and are classified as pro- and anti-apoptotic, depending on their effect on programmed cell death. Pro-apoptotic members are further subdivided into 'multidomain' ones that share the $\mathrm{BH} 1$, $\mathrm{BH} 2$ and $\mathrm{BH} 3$ modules, and the 'BH3-only' proteins that only share the $\mathrm{BH} 3$ module. The pro-apoptotic $\mathrm{BH} 3$-only proteins 'sense' the death stimuli and transduce them to mitochondria, where they activate the 'multidomain' pro-apoptotic proteins Bax and Bak, ultimately resulting in mitochondrial outer membrane permeabilization (MOMP) and cytochrome c release. With respect to the anti-apoptotic $\mathrm{Bcl}-2$ family members, two models of activity have been proposed and are the subject of intense investigations. In the first model, these proteins are endogenous inhibitors of the multidomain pro-apoptotic proteins, requiring to be antagonized by ' $\mathrm{BH} 3-$ only' molecules; in the second model, the anti-apoptotic $\mathrm{Bcl}-2$ family proteins act by sequestering $\mathrm{BH} 3$-only proteins, a possibility strongly supported by recent, elegant studies. ${ }^{9}$ However, a recent report puzzled these two models by proposing that Hax1, a purported Bcl-2-family-related protein of the mitochondrial IMS, mediates the elimination of active Bax, thereby introducing the concept that antiapoptotic Bcl-2 proteins can antagonize MOMP also from inside the mitochondria through the activation of a proteolytic cascade. $^{10}$

\footnotetext{
${ }^{1}$ Mitochondria Biology Laboratory, CRULRG, Université Laval, Quebec, QC, Canada; ${ }^{2}$ Molecular Neuropathobiology Laboratory, Cancer Research UK, London, UK; ${ }^{3}$ National Center for Biotechnology Information, NLM, NIH, Bethesda, MD, USA and ${ }^{4}$ Department of Cell Biology, Medical Biochemistry and Pathology, Faculty of Medicine, Université Laval, Quebec, QC, Canada

*Corresponding author: L Pellegrini, Mitochondria Biology Laboratory, CRULRG, Room F-6500, 2601 Ch. De la Canardiere, Quebec, QC, Canada G1J 2 G3.

Tel: + 14186635000 ext 6879; Fax: + 141866 35000; E-mail: Luca.Pellegrini@crulrg.ulaval.ca

Keywords: Parl; Hax1; rhomboids; mitochondrial stress; apoptosis; neurodegenerative disease

Abbreviations: MOMP, mitochondrial outer-membrane permeabilization; IMS, mitochondrial intermembrane space; IMM, inner mitochondrial membrane

Received 18.5.09; revised 08.7.09; accepted 09.7.09; Edited by D Vaux; published online 14.8.09
} 
To ensure the complete release of cytochrome $c$, the architecture of the mitochondrial reticulum and the ultrastructure of the organelle changes in the early stages of apoptosis. ${ }^{1-14} \mathrm{~A}$ combination of electron tomography and physiological measurements identified a pathway of cristae remodeling characterized by the widening of the narrow tubular junction and by the fusion of individual cristae. These morphological changes support the mobilization of cytochrome c from the cristae to the IMS, and eventually to the cytosol. ${ }^{15}$ Mechanistically, the mitochondrial rhomboid protease PARL and the dynamin-related GTPase Opa1, two proteins of the IMM, participate in the control of the shape and structure of the cristae and of the cristae junctions. ${ }^{16,17}$ Under steady state conditions, active Opa1 can prevent the widening of the cristae junctions by forming an oligomer that functions as a molecular staple between the adjacent membranes of the cristae. This high molecular weight complex contains both an IMM-bound form of Opa1 and an IMS-soluble one. Generation of the latter form requires the rhomboid protease PARL, whose ablation is lethal in adult mice owing to a pathology caused by excessive apoptosis in multiple tissues. ${ }^{16,17}$ A similar phenotype was recently observed in mice lacking Hax1, which also displayed reduced levels of a cleaved form of Omi/HtrA2, ${ }^{10}$ a serine protease of the IMS implicated in oxidative stress and apoptosis. ${ }^{18}$ These observations and the finding that Hax1 could be co-immunoprecipitated with PARL suggested a model in which Hax1 presents Omi/HtrA2 to PARL, to generate a form of Omi/HtrA2 that may proteolytically antagonize from the IMS active Bax during MOMP, ${ }^{10}$ thereby defining a novel anti-apoptotic Opa1-independent pathway for PARL. ${ }^{16,17,19}$

Our study shows that Hax1 is not a bona fide Bcl-2-familyrelated protein because it does not share any sequence similarity with $\mathrm{BH}$ modules, and its confidently predicted secondary structure is incompatible with the presence of $\mathrm{BH}$ modules. We further show that, in vivo, the activity of Hax1 cannot be mechanistically coupled to PARL because the two proteins are confined in different cellular compartments, and their interaction in vitro is an artifact. Hax1 has a key role in autosomal recessive severe congenital neutropenia, ${ }^{20}$ a primary immunodeficiency syndrome associated with increased apoptosis in myeloid cells; therefore, correcting the mechanism of Hax1 activity remains an outstanding question, which has to be addressed to decipher the molecular pathways that link mitochondrial stress response to apoptosis.

\section{Results}

Hax1 lacks BH modules. Hax1 was initially proposed to be a Bcl-2 family protein on the basis of purported structural similarities to $\mathrm{Bcl}-2$ family members, including the presence of $\mathrm{BH} 1$ - and $\mathrm{BH} 2$-like modules and a C-terminal transmembrane domain. ${ }^{21}$ Recent studies have embraced this notion, ${ }^{10}$ contributing to consolidate Hax1 as a member of this important family of proteins. However, our sequence analysis and structure prediction do not support the presence of any $\mathrm{BH}$ modules in Hax1. Indeed, the purported $\mathrm{BH} 1$ and $\mathrm{BH} 2$ modules, located at position $37-56$ and $74-89$, respectively, are not recognized by conserved protein domain search, even with the most relaxed threshold. Further, multiple secondary structure predictions show with full consistency that the regions of $\operatorname{Hax} 1$, which were previously aligned with the $\mathrm{BH} 1$ and $\mathrm{BH} 2$ modules, are largely disordered, whereas the bona fide $\mathrm{BH} 1$ and $\mathrm{BH} 2$ modules are stable hairpins formed by hydrophobic $\alpha$-helices. ${ }^{22,23}$ In addition, the corresponding regions of the Hax1 sequence are not well conserved even in closely related animals, such as mammals, and show no sequence conservation at all in more distantly related species (Figure 1), which would be incompatible with the key roles of these regions in the function of Hax1 in apoptosis as a $\mathrm{Bcl}-2$ protein. Our analysis shows instead that Hax1 is an $\alpha / \beta$-protein that contains a strongly predicted and relatively well-conserved, in animals, three-strand $\beta$-sheet near the C-terminus (Figure 1), a structural element absent in Bcl-2 proteins. Interestingly, Hax1 also contains a conspicuous pattern of three universally conserved aspartates embedded in a predicted disordered loop, which is suggestive of functionally important metal (possibly, calcium)-binding residues. None of these structural elements are present in any of the $\mathrm{Bcl}-2$ proteins. We conclude that it is exceedingly unlikely that Hax1 is a member of the Bcl-2 family.

Hax1 is not an integral membrane protein. At the C-terminus of Hax1, there is a strongly predicted and conserved $\mathrm{C}$-terminal $\alpha$-helix, which has been purported to constitute the transmembrane domain that anchors Hax1 to both mitochondrial membranes. ${ }^{10}$ Multiple methods of transmembrane region prediction, as well as visual inspection of Hax1 sequence for long hydrophobic stretches, indicate that Hax1 does not contain such transmembrane domain, either near the C-terminus or anywhere within the protein sequence (Figure 1). To span the lipid bilayer, an $\alpha$-helix must be composed by a minimum of about 20 amino acids, mainly hydrophobic. ${ }^{24}$ The purported transmembrane domain of Hax 1 consists in an $\alpha$-helix of 16 amino acids, 4 of which are charged and 1 is polar (RPPALDDAFSILDLFL), which could not form a transmembrane domain capable of anchoring Hax1 within a lipid bilayer. This analysis is consistent with our experimental data from alkaline and high salt extraction of heavy membranes prepared from HeLa cells, which showed that endogenous $\operatorname{Hax} 1$ is peripherally associated, but not integrated, to these membranes (Figure 2). We conclude that Hax1 is not an integral membrane protein and suggest that the conserved C-terminal domain might be functionally important in coordinating Hax1 interaction with other proteins.

Hax1 is not localized inside the mitochondria. A recent report claimed that endogenous Hax 1 is localized on the inner and on the outer mitochondrial membrane, where it is exposed to the IMS. ${ }^{10}$ However, such dual membrane localization and protein topology are at odd with those of every other known bona fide mitochondrial protein, as well as with the known mechanisms of protein import in the organelle. Hax1 does not contain cysteine residues required for IMS protein import through the MIA pathway, ${ }^{25}$ or any predictable 


SS_PRPROT
SS_JPRED
SS_PSIPRED
SS_BH1
HAX1_HUMAN
HAX1_DOG
HAX1_MOUSE
HAX1_PLATIPUS
HAX1_EROG
HAX1_DANIO
HAX1_LANCELET
HAX1_WASP
HAX1_SEA_URCHIN
SS_PRPROT
SS_JPRED
SS_PSIPRED
SS_BH2
HAX1_HUMAN
HAX1_DOG
HAX1_MOUSE
HAX1_PLATIPUS
HAX1_EROG
HAX1_DANIO
HAX1_LANCELET
HAX1_WASP
HAX1_SEA_URCHIN

SS_PRPROT
SS_JPRED
SS_PSIPRED
HAX]1_HUMAN
HAX1_DOG
HAX1_MOUSE
HAX1_PLATIPUS
HAX1_EROG
HAX1_DANIO
HAX1_LANCELET
HAX1_WASP
HAX1_SEA_URCHIN

SS_PRPROT
SS JPRED

SS_PSIPRED

HAX1_HUMAN

HAX1 DOG

HAX1 MOUSE

HAX1 PLATIPUS

HAX1 FROG

HAX1_DANIO

HAX1 LANCELET

HAX1 WASP

HAX1 SEA URCHIN cchh hhhhce

cchh hhhhec

cchh hhhhhh

MSLF-DLFRGFF

MSFF-DLFRGFF

MSVF-DLFRGFF

GSAL-TGRRKRM

MSLF-ELFRRFF

MSVF-DLFRGFF

MSLH-DLFRGLF

MPFF-EFFRNLF

MSRFDDIFDSFF
$\mathrm{CCCCCC}$
$\mathrm{CCCCCO}$
$\mathrm{CCCCCO}$

GFPGPR

GLSGPR

GFPGPR

1 GDGGTY

E-PGGR

GVPGGH

GFHGGH

GK-GPA 11 QRYRDGFRNPIWQ-----TDDDEDDISDF-SNRHPANRF----------QFRIFSD----

GR-SGF 28 SSPGSRFYSEOPH----DMDDDDNYEGP-PGROGPEPG---o--o---GGATES--0-

\begin{abstract}
cee
$\mathrm{CCCC}$

CCCChhhhhhhhhhhecCCCCCCC

$\mathrm{CCCCCCCCCC} \mathrm{CC} \quad \mathrm{CCCCCCCCC}$

$\operatorname{CCCCCCCCC} \mathrm{CCCC}$

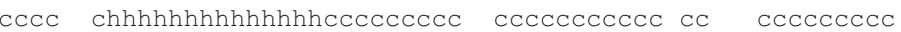

$\mathrm{CCCCCCCC}$
$\operatorname{ccccccc}$

chhhhhhhcC $\mathrm{CCCC}$

cechhhhec $\mathrm{CCCC}$

$\begin{array}{lll}\text { hhh } & \text { hhh } & \text { CCCChhhh } \\ +++ & \text { "BH2" }\end{array}$

GIRF------HDNF--GFDDLVRDFNSIFSDMGAWTLPS--HPPELPGP--E-SE----TPGERLREG---QTIRDSMLKY--PDSH -MRF------HDNE--GFDDLIRDFNNIFSEMEAWTLPS--RPSELPGPGPE-SE----TPGERRREG---QTIRDSMLKY--PDSH GMRE-------HGNE--GFDDLVRDFNS IFSEMGAWTLPS--HSPELPGP--E-SE----TPGERLREG---QTIRDSMLKY--PDSH GLHF------HDNF--GFDELVRDFNRIFSOMDTWTLPA--RRPELPGA--E-AEPG--PPGPSGSPGGRRETIRDSMLKY--PDSQ --PM-------ANVF--GFEEFERDFNELFADFGSITQ----VVPELPGVDPP-------LQRPEVPRG---KSIRDFMLKS--PDSH -ARF------EEPQ--MFGQIFRDMEEMFAGLGRFDERHGFGPRGFPS IEAPPPQEGVEKGRSGTGSG---NP IRDEMLKS--PDR--EFGGDDFGMSDMMK-HFDEMFHTFDELFRQLGTVE----FPPLSPPHRP---GVPGMEPPSSGPGHE---RSIRDRMLKE--PGA------PFEMTRFFETQMDDMMRNFFGFGNGFGNDT--NIFLPFGNENALPM--------PGENPVG---KGPRDEVLKAEVPDS-----------ENNEMNDFFKLFDDMFKSFGIAD----FPPLDVPRISPS--------SPAQPEA---KAPRDEMLKE--PDS-
\end{abstract}

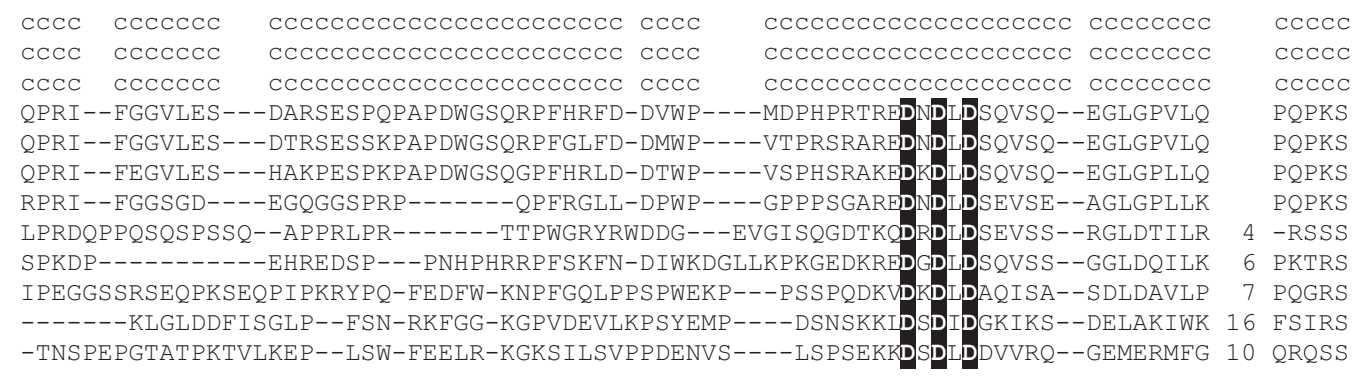
ccleeeeeeeecccceeeeeecccceeee eeec cccc ccceeeeeeeecccceeeeeeeccccceee eeee cccc cccceeeeeecccccceeeeecccceeee eeec cccc
$\mathrm{CCCCCCCC}$
cCCCC cchhhhhhhhhhhecCC $\mathrm{CCCCCCC}$ CCCCC cchhhhhhhhhhhhecC CCCCC CCChhhhhhhhhhhhcCC +++++ "TMD" ++++++
YFKSISVTKITKPDGIVEERRTVVDSEGRTETT-VTRHE--ADSS----PRGDPESP YFKSVTVTKITKPDGTVEERRTVVDSEGRTETT-VTHQE--ADGS----PRDDPESP YFKSISVTKITKPDGTVEERRTVVDSEGRRETT-VTHQE--AHDS----SRSDPDSQ YYQSVSVTTVLAPDGTVEERRTVVDSEGRTETTTVTRRG--GDDA----PAG---AP 13 RTPAL--GDATSVLDLLLGRWFRPR FFQSVSVSKIVRPDGTIEERRTVRDGQGNSSTT-VTVQR--GDEILSSETQDGPQGP 18 SPPDL--SDSQTLLSRILQKWFSQR FFKSVSVTKVVRPDGTVEERRTVRDGEGNEETT-VTISERPGGQDRPVLDQSGPLMP GGSDM--QDDFSMFSKFF-RGFR-S FFKSITTTTIRGPDGKVEQRRTVRDGSGNEETV-VTRND--GDQTHTVVTKRDPSGR 32 GQPRLPDDNTASIFYNLFGSWFGGK FGSSVSTQIVRRPDGSMEERRTVRDSDGNEEIK-ITRQI--GDKMHTIITKRAKDGS 18 TPPED-NRSDGFPWHKFFGPNPK-I YSRSISIQTIRRPDGTSETRRTERDGQGNVTTT-VTTGP--DDKPSPGSTEPRRMEP 35 GRQEG-DENDDSMYKKFFGSWFK-P

Figure 1 Hax1 does not contain BH1 or BH2 modules, or a transmembrane domain. Multiple alignment of selected Hax1 sequences from diverse animals. The numbers between aligned blocks indicate poorly conserved sequence segments that are not shown. Secondary structure (SS) predicted with three methods is shown above the alignment. c, random coil (disordered structure); $\mathrm{h}, \alpha$-helix; e, $\beta$-strand (extended conformation). Amino acid residues that are conserved in all aligned sequences are shown in bold type, and the three invariant aspartates that comprise a putative metal-binding site are shown by reverse shading. The positions of purported BH1 and BH2 modules are shown above the respective regions of the alignment using the alignment from Sharp et al. ${ }^{21}$ for $\mathrm{BH} 1$, and arbitrarily centering the alignment on a conserved hydrophobic residue for $\mathrm{BH}$ 2. 'SS_BH' denotes the consensus secondary structure motifs of $\mathrm{BH} 1$ and $\mathrm{BH} 2$ modules derived from multiple crystal and NMR structures of Bcl-2-family proteins. $^{23}$ The position of the transmembrane domain (TMD) predicted in Sharp et al., ${ }^{21}$ but not in our analysis, is indicated in the C-terminal block of the alignment

mitochondrial import peptide. Further, recent extensive proteomic studies do not list Hax1 in the compendium of mitochondrial proteins. ${ }^{26,27}$ Consistent with these findings, our data show that heavy membranes contain Hax1, but that the protein is absent in highly purified, intact mouse liver mitochondria preparations (Figure 3). We conclude that
Hax1 is not imported inside the mitochondria, although it might be peripherally associated to the organelle.

PARL association to Hax1 is unspecific. PARL is a 7-transmembrane domain-containing protein of the IMM. ${ }^{28,29}$ Topological studies have shown that the 


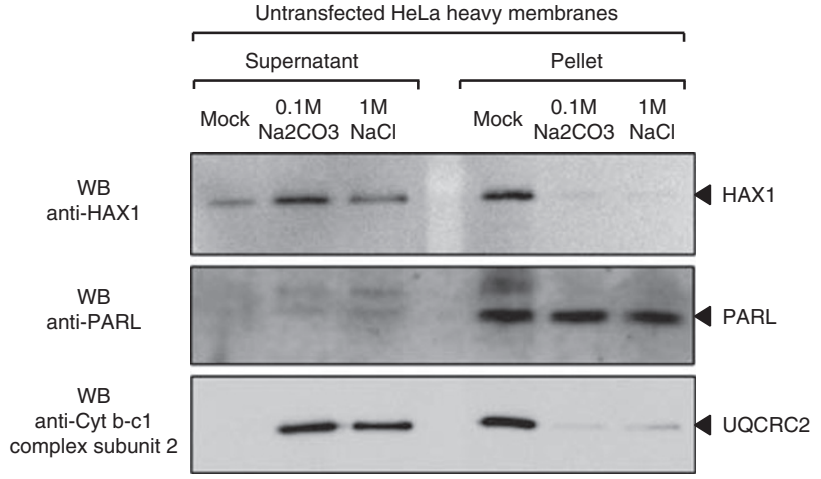

Figure 2 Hax1 is not an integral membrane protein. Alkaline extraction of heavy membranes isolated from HeLa cells $(200 \mu \mathrm{g})$; whereas membrane-associated proteins and proteins associated to membrane-bound proteins (e.g. UQCRC2) are solubilized in the supernatant, integral membrane proteins like PARL are recovered in the membrane pellet. Lack of Hax1 integration in heavy membranes is consistent with our computational analysis (Figure 1), which does not predict any potential transmembrane domain

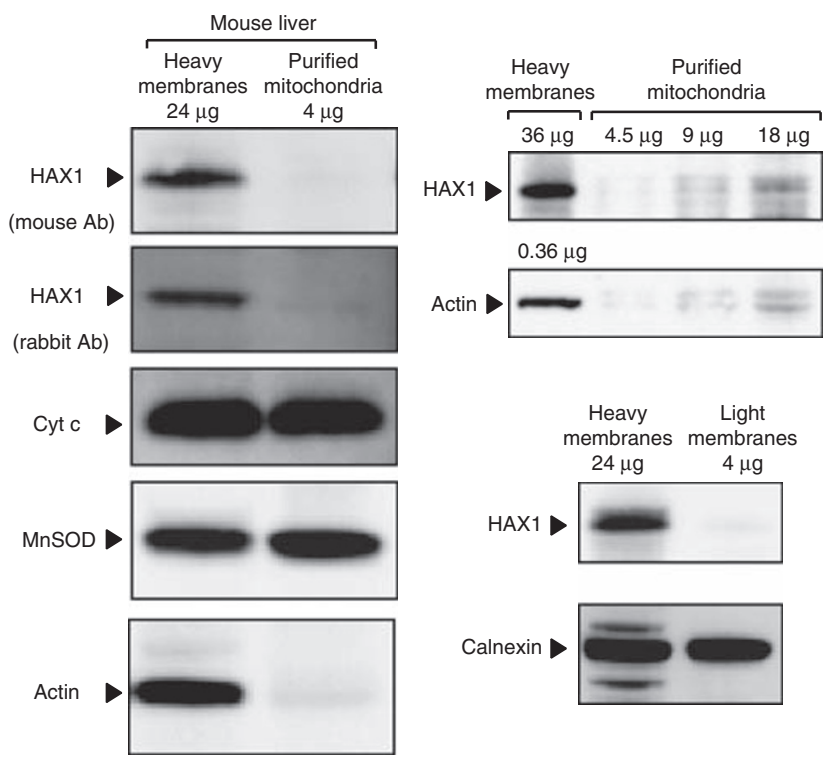

Figure 3 Hax 1 is not imported in mitochondria but is peripherally associated to heavy membranes. Immunoblot analysis of Percoll-purified fractions of mouse livers. In this preparation, lack of cross-contaminating organelles was assayed by electron microscopy (not shown); inner and outer mitochondrial membrane integrity was tested for presence of diffusible proteins of the IMS (cytochrome c) and mitochondrial matrix (MnSOD); purity from membranes-associated cytosolic proteins by the absence of actin. The left panel shows that, on normalization for cytochrome $c$ and MnSOD, endogenous Hax1 is detected in heavy membranes but not purified mitochondria, a finding consistent with lack of Hax1 in the human and murine mitochondrial proteome ${ }^{26}$

$\mathrm{N}$-terminus of the protease is exposed to the matrix and the C-terminus to the $I_{M S}{ }^{29}$ (Figure 4a). PARL contains three loops exposed to the IMS: one large Loop-A that is functionally dispensable for PARL proteolytic activity (Figure $4 a$ and $b)^{29}$ and seems to coordinate the interaction with OPA $1,{ }^{17}$ and two very small loops that are a part of the rhomboid domain. ${ }^{28,30}$
Recently, co-immunoprecipitation studies were used to propose a mechanism in which Hax1 interaction with PARL allows presentation of the serine protease Omi/HtrA2 to the rhomboid protease to generate a cleaved active form of Omi/HtrA2 in the IMS. ${ }^{10}$ As this model is incompatible with the notion that Hax1 is not targeted to the IMS (Figure 3; Pagliarini et $a .^{26}$ and Yamada et $a{ }^{27}{ }^{27}$ ), we investigated the unspecific nature of the reported PARL/Hax1 interaction ${ }^{10}$ by cotransfecting HEK293 cells with constructs expressing wildtype Hax 1 and mutant forms of PARL that lack mitochondrial import. Data showed that Hax1 could be co-immunoprecipitated with PARL irrespective of the fact that the latter was neither properly targeted nor folded in the IMM (data not shown), suggesting that Hax1 association is not mediated by IMS domain(s) of PARL but, rather, by unspecific hydrophobic interaction between the two misfolded proteins. To address this possibility, we deleted the only two IMS domains of PARL that could potentially bind to Hax1 and confirmed their dispensability for co-immunoprecipitating Hax1 (Figure 4b). Further, we excluded their role as bona fide IMS Hax1-binding domain by means of a mammalian two-hybrid system (Figure 4c).

To further validate the unspecific nature of the PARL/Hax1 association, we tested whether Hax 1 co-immunoprecipitation with PARL occurs after cellular lysis by mixing (i) lysates of cells that were independently transfected with constructs expressing PARL and Hax1 (Figure 5a) and (ii) lysates of heavy membranes isolated from Parl-/- and Hax1-/- MEFs (Figure 5b). In both cases, PARL/Hax1 association could be recreated, supporting the conclusion that the interaction between these two proteins is an in vitro artifact that occurs post lysis through unspecific hydrophobic interactions.

PARL activity does not require Hax1. Although some types of proteases require substrate presentation by an accessory protein, rhomboids do not seem to need one. ${ }^{31,32}$ The recently proposed Hax1/PARL model assigns a PARL substrate-presenting function to Hax $1 .{ }^{10,33}$ However, this seems to be an unlikely possibility because loss of Hax1 function only partially reduces the expression of a cleaved form of Omi/HtrA2. ${ }^{10}$ Further, genetic ablation of Hax1 compromises neither Opa1 cleavage ${ }^{10}$ nor PARL cleavage (Figure 6), an $\mathrm{N}$-terminal processing that requires PARL activity supplied in trans. ${ }^{34}$ Together with the non-IMS localization of Hax1 (Figure 3; Pagliarini et al. ${ }^{26}$ and Yamada et al. ${ }^{27}$ ), these evidences indicate that the protein is not a PARL substrate-presenting protein and that PARL rhomboid activity does not require Hax1.

\section{Discussion}

This study arises from a recent report that addressed the role of Hax1 in mediating the processing of the mitochondrial stress-related Omi/HtrA2 protease to allow the survival of lymphocytes and neurons. ${ }^{10}$ In this study, cell-type sensitivity to pro-apoptotic stimuli was proposed to be governed by the formation of a complex between Hax1, a purported $\mathrm{Bcl}-2$ family-related protein, and PARL, a mitochondrial rhomboid protease implicated in apoptosis and mitochondria dynamics regulation. ${ }^{17,29}$ Mechanistically, Hax1/PARL 
a

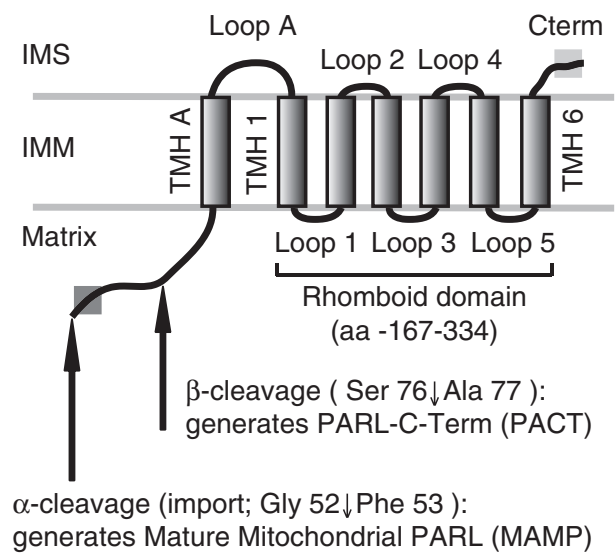

Epitope of anti-PARL-Nterm

$\mathrm{N}$-terminus (P $\beta$ domain) : aa 53-100

TMH A : aa 101-118

Loop A : aa 119-166

TMH 1 : aa 167-185

Loop 1 : aa 186-217

Loop 2 : aa $241-243$

Loop 3 : aa 264-273

Loop 4 : aa 292-298

Loop 5 : aa $319-330$

TMH 6 : aa $331-350$

C-terminus : aa 351-379

Epitope of anti-PARL-Cterm

b

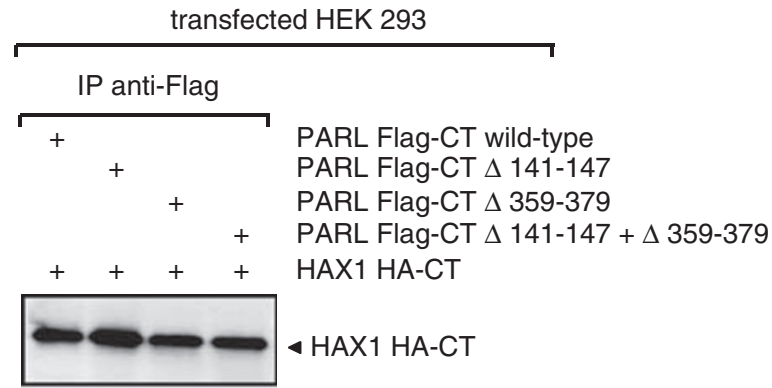

WB anti-HA

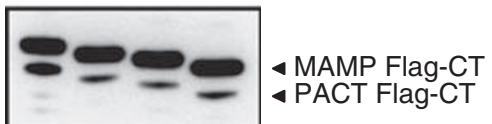

WB anti-Flag

C $\mathrm{pACT}-\mathrm{HAX} 1+\mathrm{pBIND}-\mathrm{LD}$

pACT-MyoD + pBIND-PARL C-term

pACT-MyoD + pBIND-LD

pACT-HAX1 + pBIND-PARL Loop A

pACT-HAX1 + pBIND-PARL N-term

pACT-HAX1 + pBIND-PARL C-term

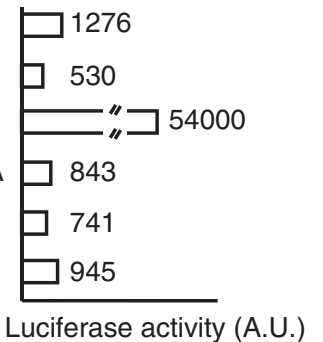

Figure 4 PARL does not have a domain that coordinates interaction to Hax1 in the IMS. (a) Schematic representation of the topology and domain composition of PARL. ${ }^{28,30}$ (b) Co-immunoprecipitation of Hax1 with mutant forms of PARL in which the IMS domains (loop-A and C-terminus) have been deleted. Note that PACT formation in indicates that the mutant proteins are correctly imported and folded in the inner membrane. (c) Mammalian two-hybrid assays fails to identify an IMS domain in PARL that could mediate interaction to Hax1. For a positive control reaction, pBIND-LD and pACT-MyoD control vectors are cotransfected along with pG5luc. Values, expressed as arbitrary units (A.U.), indicate mean of three independent experiments done in triplicates

association in the IMS was reported to allow the recruitment and presentation of Omi/HtrA2 to PARL, ${ }^{10,33}$ to generate a cleaved active form of Omi/HtrA2 that, in turn, could antagonize MOMP from the IMS through proteolytic elimination of active $\mathrm{Bax},{ }^{10} \mathrm{a}$ concept that, however, is at odd with the notion that active Bax does not expose to the IMS, a cleavable domain. ${ }^{6}$

For Hax1 to recruit and present Omi/HtrA2 to PARL, the protein must be localized within the IMS. However, although we observed an association of Hax1 with the heavy membrane fraction, we did not detect Hax1 in Percoll-purified mouse liver mitochondria (Figure 3 ), a finding consistent with the absence of $\mathrm{Hax} 1$ from the mitochondrial proteome, ${ }^{26,27}$ as well as with the lack of a predictable mitochondrial targeting peptide or amino acid signature in animal orthologs of this protein (Figure 1 and data not shown). The association of Hax1 with heavy membranes is peripheral, as it was removed by alkaline and high salt extraction (Figure 2). Thus, contrary to previous reports, ${ }^{10,35}$ we found that endogenous Hax 1 is neither resident inside the mitochondria nor anchored to the 


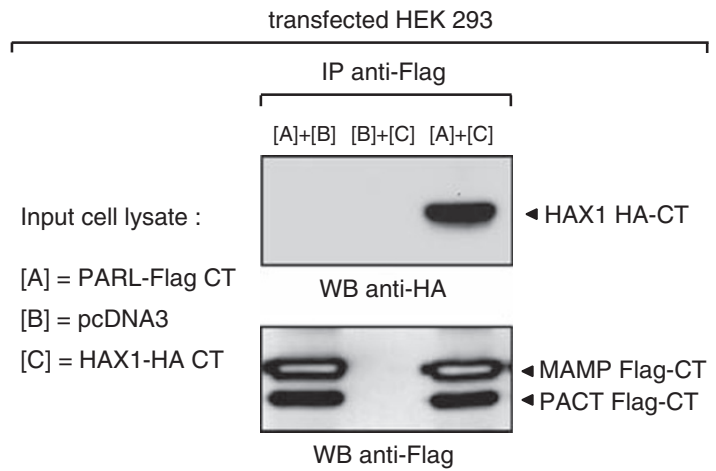

b

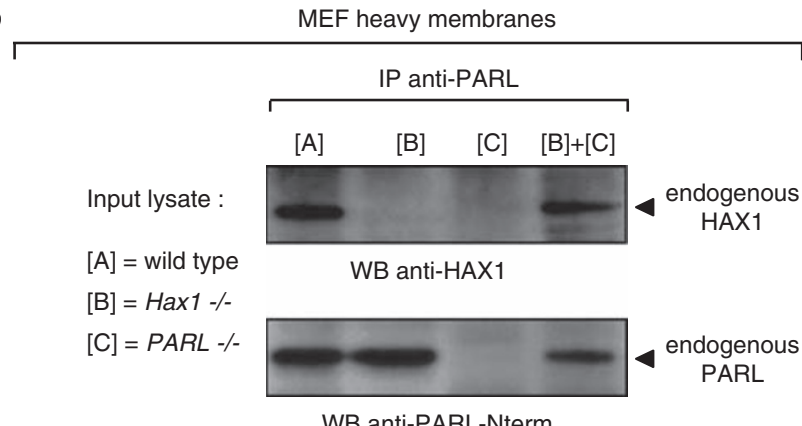

Figure 5 Hax1 binding to PARL is unspecific. (a) In vitro reconstitution of PARL/ Hax1 complex by mixing lysates of HEK293 cells transfected with a construct expressing PARL-Flag-CT or Hax-HA-CT. (b) In vitro reconstitution of PARL/Hax1 complex by mixing lysates of heavy membranes $(150 \mu \mathrm{g}$ of proteins per genotype used) isolated from MEFs $+/+$, Hax $1-/-$ or Parl -/-
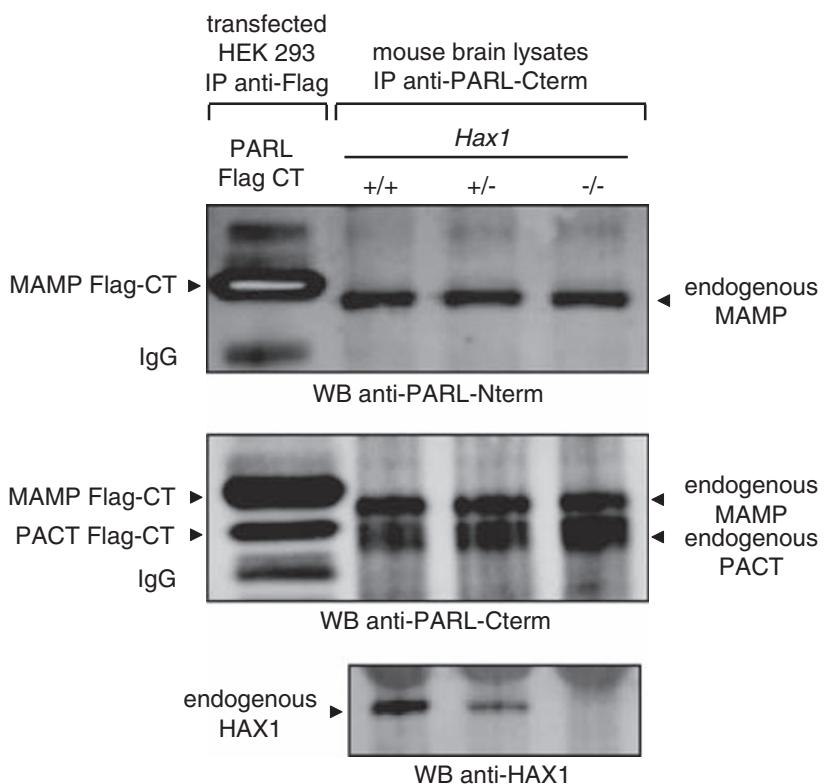

Figure 6 PARL proteolytic activity does not require Hax1. The upper panel shows that the level of expression of the mitochondrial mature form of PARL, MAMP, is not altered in any of the indicated genotypes. The middle panel indicates that genetic ablation of Hax1 does not impair the generation of PACT, a shorter form of MAMP that requires PARL activity supplied in trans. ${ }^{34}$ The lower panel shows the expression levels of Hax1 in mouse Hax1 $+/+,+/-$ and $-/-$ brain lysates membranes of the organelle. Although our findings cannot exclude the possibility that a subtle amount of Hax1 could be targeted inside the mitochondria, they nevertheless indicate that the loss of the heavy membrane-associated form of Hax1 is likely responsible for the lethal phenotype displayed by Hax $1-/-$ mice, as well as for the reduced levels of processed Omi/HtrA2. ${ }^{10}$

In this study, we tested the specificity of the interaction between Hax1 and PARL, a highly hydrophobic protein of the IMM. $^{28,29}$ Consistent with previous reports, ${ }^{10}$ we also observed that Hax1 can be co-immunoprecipitated with PARL, but not vice versa (not shown). However, the specificity of this association is dubious at best. Mixing lysates of cells that were independently transfected with constructs expressing PARL-Flag-CT and Hax1-HA-CT reconstituted PARL/ Hax1 interaction (Figure 5a); similarly, the interaction of endogenous PARL and Hax1 could be recreated by mixing lysates of heavy membranes isolated from Parl-/- and Hax1-/- MEFs (Figure 5b). Furthermore, we could not identify any domain within PARL that could mediate interaction to Hax1 (Figure 4). Together, these results suggest that Hax1 does not specifically bind PARL and thus it cannot present a substrate to PARL. ${ }^{10,33}$ This conclusion is consistent with the observation that genetic ablation of Hax1 does not fully abolish Omi/HtrA2 processing, ${ }^{10}$ it is not required for the cleavage of OPA $1^{10}$ and it does not affect PARL $\beta$-cleavage (Figure 6 ), which is self-regulated. ${ }^{34}$

Whether mammalian PARL cleaves Omi/HtrA2, a mitochondrial stress response serine protease, ${ }^{18,36}$ remains to be shown, but in the light of the data presented here, a direct role of Hax1 in this processing can be ruled out. Given the peripheral association of Hax1 to heavy membranes as well as its possible calcium-binding capacity (Figure 1) and its interaction with Phospholamban, ${ }^{37}$ a sarcoplasmic reticulum protein and a key regulator of $\mathrm{Ca}^{2+}$ homeostasis, the reduced level of processed Omi/HtrA2 observed in Hax1-/- cells could be explained by the defects in calcium signaling and homeostasis. ${ }^{38-43}$ Accordingly, the phenotype of the Hax1-/- mouse could be explained by multiorganellar failure due to defective calcium homeostasis. Thus, our study re-opens the question of whether and how the organelle governs PARL activity to regulate mitochondrial stress, a process that can trigger mitochondrial dysfunctions that are central to the etiology of cancer ${ }^{44-46}$ and multiple neurodegenerative disorders, including Parkinson's disease. ${ }^{47,48}$

Our study showed that Hax1 is not an IMS protein containing $\mathrm{BH}$ modules, thereby ruling out a model in which $\mathrm{Bcl}-2$ proteins mediate the elimination of active Bax from inside the organelle during MOMP. Identification of Bcl-2 family-related proteins is a challenging task because some members of this family show no conserved sequence motifs (at least, not statistically significant ones) ${ }^{49}$ and are recognized solely on the basis of structural similarity to bona fide Bcl-2-family proteins. ${ }^{23}$ However, Hax1 does not meet even the most liberal criteria for the presence of $\mathrm{BH}$ modules. First, the secondary structure of Hax1 that we confidently and consistently predicted with three computational methods is incompatible with the presence of such modules and, second, the sequences of Hax1 that were previously aligned with $\mathrm{BH} 1$ and $\mathrm{BH} 2$ domains are very poorly conserved during the 
evolution of Hax1, an observation that effectively rules out a key role of these regions in Hax1 function and that is in agreement with reports recently published by other groups. ${ }^{50}$ In conclusion, although it could be formally argued that only the crystal structure of Hax1 can put the hypothesis that Hax1 is a member of the Bcl-2 family to final rest, the analysis reported here makes this possibility exceedingly unlikely.

\section{Materials and Methods}

Computational analysis of protein sequences. Hax1 orthologs from all the sequenced animal genomes were detected by searching the Genpept database using the PSI-BLAST program, ${ }^{51}$ and a multiple alignment of a selected set of diverse sequences was constructed using the T-Coffee program. ${ }^{52}$ Secondary structure (SS) prediction was performed using the program PSIPRED, ${ }^{53}$ JPRED $^{54}$ and PredictProtein (PRPROT). ${ }^{55}$ The search for conserved protein domains was performed using the RPS-BLAST program and the Conserved Domain Database. ${ }^{56,57}$ Mitochondrial import prediction was done using the MitoProt II program. ${ }^{58}$ Transmembrane domains were predicted using PredictProtein, ${ }^{55}$ as well as TMHMM ${ }^{59}$ and TMPred. ${ }^{60}$

Mitochondria purification. Mitochondria were purified as described. ${ }^{41}$ Briefly, mouse livers were washed once with PBS, suspended in isolation buffer (200 mM sucrose, $1 \mathrm{mM}$ EGTA-Tris and $10 \mathrm{mM}$ Tris-MOPS, pH 7.4), and then disrupted by dounce homogenization on ice. The homogenate was spun at $800 \times g$ for $10 \mathrm{~min}$ at $4^{\circ} \mathrm{C}$; the supernatant was recovered and further centrifuged for $10 \mathrm{~min}$ at $8000 \times g$ at $4^{\circ} \mathrm{C}$. The resulting pellet (mitochondrial fraction) was collected, whereas the supernatant was further spun for $30 \mathrm{~min}$ at $100000 \times \mathrm{g}$ at $4^{\circ} \mathrm{C}$. The resulting pellet (light membrane fraction) and supernatant (cytosolic fraction) were spun again at $100000 \times g$ to further purify the fractions. The mitochondrial fraction was purified further by centrifuging twice at $8000 \times g$ for $10 \mathrm{~min}$ at $4^{\circ} \mathrm{C}$. The obtained pellet was purified by centrifugation at $95000 \times g$ for $30 \mathrm{~min}$ on a $30 \%$ Percoll gradient in isolation buffer. The mitochondrial layer was washed free of Percoll and resuspended in isolation buffer. Subcellular fractions were also obtained by differential centrifugation from mouse liver and MEFs as described. ${ }^{61}$ Protein concentration was determined and the indicated amounts of protein were separated by SDS-PAGE and immunoblotted.

Alkaline extraction. Heavy membranes prepared from HeLa cells were diluted to a final concentration of $1 \mathrm{mg} / \mathrm{ml}$ in $20 \mathrm{mM} \mathrm{HEPES} / \mathrm{KOH}(\mathrm{pH} 7.4)$ (hyposmotic buffer). After the addition of an equal volume of freshly prepared $0.2 \mathrm{M}$ sodium carbonate $(\mathrm{pH} 11.5)$, samples were incubated for $30 \mathrm{~min}$ at $4^{\circ} \mathrm{C}$. The membrane and soluble fractions were separated by ultracentrifugation at $100000 \times g$ for $30 \mathrm{~min}$ at $4^{\circ} \mathrm{C}$.

Cell culture and transfection. HEK 293T and HeLa cells were purchased from the American Type Culture Collection (Manassas, VA, USA). All cell types used in this study were maintained under standard cell culture conditions. Cells were transfected at $40 \%$ confluence with FuGENE 6 (Roche, Mannheim, Germany). Hax1-/- MEFs and brains were kindly provided by Dr. JN Ihle; Parl-/- MEFs by B De Strooper.

Cloning and mutagenesis. The pcDNA3 vector was used to express the human PARL and mouse Hax1 protein in mammalian cells. Mutants were obtained by site-directed mutagenesis (Clontech, Mountain View, CA, USA). For the mammalian two-hybrid assay, the Checkmate System and vectors (pBind, pACT, pG5luc) were used (Promega, Madison, WI, USA). The identity of every construct and mutant was confirmed by DNA sequencing.

Co-immunoprecipitations. Transfected HeLa cells were lysed in STEN buffer; heavy membranes in CHAPS buffer. Immunoprecipitations were conducted as described. ${ }^{10,29}$

Antibodies. Rabbit anti-PARL; ${ }^{29,34}$ rabbit anti-MnSOD (Stressgen, Ann Arbor, MI, USA; 1: 1000); mouse anti-UQCRC2 (clone 13G12, Molecular Probes, CA, USA; 1 : 3000); mouse anti-HA (Roche); mouse anti-Flag M2 (Sigma, St Louis, MO, USA); mouse anti-actin (clone C4, Cedarlane, Hornby, ON, Canada; 1:1000); mouse anti-cytochrome $\mathrm{c}$ (clone 7H8.2C12, BD Pharmingen, Mississauga, ON,
Canada; 1 : 1000); mouse anti-Hax1 (clone-52, BD Biosciences, Mississauga, ON Canada; $1: 250$ ) and rabbit anti-calnexin (Stressgen, Ann Arbor, MI, USA; $1: 4000$ ). The polyclonal rabbit anti-Hax1 antibody was raised against peptides encompassing amino acid 126-142 and 201-217 of mouse Hax1 (1:500).

Acknowledgements. This study was supported by a grant from the Canadian Institutes of Health Research to LP (MOP-82718). DVJ is a CIHR Banting and Best Canada Graduate Scholar. LP is a FRSQ Chercheur-Boursier Senior scholar.

1. Henry-Mowatt J, Dive C, Martinou JC, James D. Role of mitochondrial membrane permeabilization in apoptosis and cancer. Oncogene 2004; 23: 2850-2860.

2. Bernardi $P$, Petronilli V, Di Lisa F, Forte $M$. A mitochondrial perspective on cell death. Trends Biochem Sci 2001; 26: 112-117.

3. Martinou JC, Youle RJ. Which came first, the cytochrome $\mathrm{c}$ release or the mitochondrial fission? Cell Death Differ 2006; 13: 1291-1295.

4. Jourdain A, Martinou JC. Mitochondrial outer-membrane permeabilization and remodelling in apoptosis. Int J Biochem Cell Biol 2009, doi:10.1016/.j.biocel.2009.05.001.

5. Scorrano L. Opening the doors to cytochrome $\mathrm{c}$ : changes in mitochondrial shape and apoptosis. Int J Biochem Cell Biol 2009, doi:10.1016/j.biocel.2009.04.016.

6. Youle RJ, Strasser A. The BCL-2 protein family: opposing activities that mediate cell death. Nat Rev Mol Cell Biol 2008; 9: 47-59.

7. Aravind L, Dixit VM, Koonin EV. Apoptotic molecular machinery: vastly increased complexity in vertebrates revealed by genome comparisons. Science 2001; 291: 1279-1284.

8. Germain M, Shore GC. Cellular distribution of Bcl-2 family proteins. Sci STKE 2003; 2003: pe10.

9. Lovell JF, Billen LP, Bindner S, Shamas-Din A, Fradin C, Leber B et al. Membrane binding by tBid initiates an ordered series of events culminating in membrane permeabilization by Bax. Cell 2008; 135: 1074-1084.

10. Chao JR, Parganas E, Boyd K, Hong CY, Opferman JT, Ihle JN. Hax1-mediated processing of $\mathrm{HtrA} 2$ by Parl allows survival of lymphocytes and neurons. Nature 2008; 452 : 98-102.

11. Martinou I, Desagher S, Eskes R, Antonsson B, André E, Fakan S et al. The release of cytochrome $\mathrm{c}$ from mitochondria during apoptosis of NGF-deprived sympathetic neurons is a reversible event. J Cell Biol 1999; 144: 883-889.

12. Frank S, Gaume B, Bergmann-Leitner ES, Leitner WW, Robert EG, Catez F et al. The role of dynamin-related protein 1, a mediator of mitochondrial fission, in apoptosis. Dev Cell 2001; 1: 515-525.

13. James DI, Martinou JC. Mitochondrial dynamics and apoptosis: a painful separation. Dev Cell 2008; 15: 341-343.

14. Herzig S, Martinou JC. Mitochondrial dynamics: to be in good shape to survive. Curr Mol Med 2008; 8: 131-137.

15. Scorrano L, Ashiya M, Buttle K, Weiler S, Oakes SA, Mannella CA et al. A distinct pathway remodels mitochondrial cristae and mobilizes cytochrome $\mathrm{c}$ during apoptosis. Dev Cell 2002; 2: 55-67.

16. Frezza C, Cipolat S, Martins de Brito O, Micaroni M, Beznoussenko GV, Rudka T et al. OPA1 controls apoptotic cristae remodeling independently from mitochondrial fusion. Cell 2006; 126: 177-189.

17. Cipolat S, Rudka T, Hartmann D, Costa V, Serneels L, Craessaerts K et al. Mitochondria rhomboid PARL regulates cytochrome $c$ release during apoptosis via OPA1-dependent cristae remodeling. Cell 2006; 126: 163-175.

18. Vande Walle L, Lamkanfi M, Vandenabeele $P$. The mitochondrial serine protease $\mathrm{Htr} A 2$ Omi: an overview. Cell Death Differ 2008; 15: 453-460.

19. Pellegrini L, Scorrano L. A cut short to death: Parl and Opa1 in the regulation of mitochondrial morphology and apoptosis. Cell Death Differ 2007; 14: 1275-1284.

20. Klein C, Grudzien M, Appaswamy G, Germeshausen M, Sandrock I, Schäffer AA et al. HAX1 deficiency causes autosomal recessive severe congenital neutropenia (Kostmann disease). Nat Genet 2007; 39: 86-92.

21. Sharp TV, Wang HW, Koumi A, Hollyman D, Endo Y, Ye H et al. K15 protein of Kaposi's sarcoma-associated herpesvirus is latently expressed and binds to $\mathrm{HAX}-1$, a protein with antiapoptotic function. J Virol 2002; 76: 802-816.

22. Feng $Y$, Zhang $L, H u T$, Shen $X$, Ding J, Chen $K$ et al. A conserved hydrophobic core at $\mathrm{Bcl}-\mathrm{x}(\mathrm{L})$ mediates its structural stability and binding affinity with $\mathrm{BH} 3$-domain peptide of pro-apoptotic protein. Arch Biochem Biophys 2009; 484: 46-54.

23. Petros AM, Olejniczak ET, Fesik SW. Structural biology of the Bcl-2 family of proteins. Biochim Biophys Acta 2004; 1644: 83-94.

24. White SH, von heijne G. Transmembrane helices before, during, and after insertion. Curr Opin Struct Biol 2005; 15: 378-386.

25. Stojanovski D, Müller JM, Milenkovic D, Guiard B, Pfanner N, Chacinska A. The MIA system for protein import into the mitochondrial intermembrane space. Biochim Biophys Acta 2008; 1783: 610-617.

26. Pagliarini DJ, Calvo SE, Chang B, Sheth SA, Vafai SB, Ong SE et al. A mitochondrial protein compendium elucidates complex I disease biology. Cell 2008; 134: 112-123.

27. Yamada A, Yamamoto T, Yamazaki N, Yamashita K, Kataoka M, Nagata T et al. Differential permeabilization effects of $\mathrm{Ca} 2+$ and valinomycin on the inner and outer 
mitochondrial membranes as revealed by proteomics analysis of proteins released from mitochondria. Mol Cell Proteomics 2009; 8: 1265-1277.

28. Koonin EV, Makarova KS, Rogozin IB, Davidovic L, Letellier MC, Pellegrini L. The rhomboids: a nearly ubiquitous family of intramembrane serine proteases that probably evolved by multiple ancient horizontal gene transfers. Genome Biol 2003; 4: R19.

29. Jeyaraju DV, Xu L, Letellier MC, Bandaru S, Zunino R, Berg EA et al. Phosphorylation and cleavage of presenilin-associated rhomboid-like protein (PARL) promotes changes in mitochondrial morphology. Proc Natl Acad Sci USA 2006; 103: 18562-18567.

30. Lemberg MK, Freeman M. Functional and evolutionary implications of enhanced genomic analysis of rhomboid intramembrane proteases. Genome Res 2007; 17: 1634-1646.

31. Urban S, Wolfe MS. Reconstitution of intramembrane proteolysis in vitro reveals that pure rhomboid is sufficient for catalysis and specificity. Proc Natl Acad Sci USA 2005; 102 : 1883-1888.

32. Urban S. Rhomboid proteins: conserved membrane proteases with divergent biological functions. Genes Dev 2006; 20: 3054-3068.

33. Freeman M. Rhomboid proteases and their biological functions. Annu Rev Genet 2008; 42 191-210.

34. Sík A, Passer BJ, Koonin EV, Pellegrini L. Self-regulated cleavage of the mitochondria intramembrane-cleaving protease PARL yields Pbeta, a nuclear-targeted peptide. J Biol Chem 2004; 279: 15323-15329.

35. Kasashima K, Ohta E, Kagawa $\mathrm{Y}$, Endo H. Mitochondrial functions and estrogen receptordependent nuclear translocation of pleiotropic human prohibitin 2. J Biol Chem 2006; 281: 36401-36410.

36. Moisoi N, Klupsch K, Fedele V, East P, Sharma S, Renton A et al. Mitochondria dysfunction triggered by loss of $\mathrm{HtrA} 2$ results in the activation of a brain-specific transcriptional stress response. Cell Death Differ 2009; 16: 449-464.

37. Vafiadaki $E$, Sanoudou D, Arvanitis DA, Catino DH, Kranias EG, KontrogiannKonstantopoulos A. Phospholamban interacts with HAX-1, a mitochondrial protein with anti-apoptotic function. $J$ Mol Biol 2007; 367: 65-79.

38. Giacomello M, Drago I, Pizzo P, Pozzan T. Mitochondrial Ca2+ as a key regulator of cell life and death. Cell Death Differ 2007; 14: 1267-1274.

39. Pizzo P, Pozzan T. Mitochondria-endoplasmic reticulum choreography: structure and signaling dynamics. Trends Cell Biol 2007; 17: 511-517.

40. Szabadkai G, Simoni AM, Bianchi K, De Stefani D, Leo S, Wieckowski MR et a. Mitochondrial dynamics and $\mathrm{Ca}^{2+}$ signaling. Biochim Biophys Acta 2006; 1763: 442-449.

41. de Brito OM, Scorrano L. Mitofusin 2 tethers endoplasmic reticulum to mitochondria. Nature 2008; 456: 605-610.

42. Rimessi A, Giorgi $C$, Pinton $P$, Rizzuto $R$. The versatility of mitochondrial calcium signals: from stimulation of cell metabolism to induction of cell death. Biochim Biophys Acta 2008; 1777: 808-816.
43. Jeyaraju DV, Cisbani G, Pellegrini L. Calcium regulation of mitochondria motility and morphology. Biochim Biophys Acta 2008, doi:10.1016/j.bbabio.2008.12.005

44. Gottlieb E, Tomlinson IP. Mitochondrial tumour suppressors: a genetic and biochemical update. Nat Rev Cancer 2005; 5: 857-866.

45. King A, Selak MA, Gottlieb E. Succinate dehydrogenase and fumarate hydratase: linking mitochondrial dysfunction and cancer. Oncogene 2006; 25: 4675-4682.

46. Frezza C, Gottlieb E. Mitochondria in cancer: not just innocent bystanders. Semin Cancer Biol 2009; 19: 4-11.

47. Schapira AH. Mitochondrial dysfunction in Parkinson's disease. Cell Death Differ 2007; 14: 1261-1266.

48. Narendra D, Tanaka A, Suen DF, Youle RJ. Parkin is recruited selectively to impaired mitochondria and promotes their autophagy. J Cell Biol 2008; 183: 795-803.

49. Koonin EV, Aravind L. Origin and evolution of eukaryotic apoptosis: the bacterial connection. Cell Death Differ 2002; 9: 394-404.

50. Fadeel B, Grzybowska E. HAX-1: a multifunctional protein with emerging roles in human disease. Biochim Biophys Acta 2009, doi:10.1016/j.bbagen.2009.06.004

51. Altschul SF, Madden TL, Schäffer AA, Zhang J, Zhang Z, Miller W et al. Gapped BLAST and PSI-BLAST: a new generation of protein database search programs. Nucleic Acids Res 1997; 25: 3389-3402.

52. Notredame C, Higgins DG, Heringa J. T-Coffee: A novel method for fast and accurate multiple sequence alignment. J Mol Biol 2000; 302: 205-217.

53. McGuffin LJ, Bryson K, Jones DT. The PSIPRED protein structure prediction server. Bioinformatics 2000; 16: 404-405.

54. Cole C, Barber JD, Barton GJ. The Jpred 3 secondary structure prediction server. Nucleic Acids Res 2008; 36: W197-W201.

55. Rost B, Yachdav G, Liu J. The PredictProtein server. Nucleic Acids Res 2004; 32 W321-W326.

56. Marchler-Bauer A, Anderson JB, DeWeese-Scott C, Fedorova ND, Geer LY, He S et al. CDD: a curated Entrez database of conserved domain alignments. Nucleic Acids Res 2003; 31: 383-387.

57. Marchler-Bauer A, Bryant SH. CD-Search: protein domain annotations on the fly. Nucleic Acids Res 2004; 32: W327-W331.

58. Claros MG, Vincens $P$. Computational method to predict mitochondrially imported proteins and their targeting sequences. Eur J Biochem 1996; 241: 779-786.

59. Sonnhammer EL, von Heijne G, Krogh A. A hidden Markov model for predicting transmembrane helices in protein sequences. Proc Int Conf Intell Syst Mol Biol 1998; 6: 175-182.

60. Hofmann K, Stoffel W. TMbase - A database of membrane spanning proteins segments. Biol Chem Hoppe-Seyler 1993; 374: 166.

61. Frezza $\mathrm{C}$, Cipolat $\mathrm{S}$, Scorrano L. Organelle isolation: functional mitochondria from mouse liver, muscle and cultured fibroblasts. Nat Protoc 2007; 2: 287-295. 